\title{
Get a Sample for a Discount Sampling-Based XML Data Pricing
}

\author{
Ruiming Tang ${ }^{1}$, Antoine Amarilli ${ }^{2}$, Pierre Senellart ${ }^{2}$, and Stéphane Bressan ${ }^{1}$ \\ 1 National University of Singapore, Singapore \\ \{tangruiming, steph $\}$ @nus .edu.sg \\ 2 Institut Mines-Télécom; Télécom ParisTech; CNRS LTCI. Paris, France \\ \{antoine.amarilli,pierre.senellart\}@telecom-paristech.fr
}

\begin{abstract}
While price and data quality should define the major tradeoff for consumers in data markets, prices are usually prescribed by vendors and data quality is not negotiable. In this paper we study a model where data quality can be traded for a discount. We focus on the case of XML documents and consider completeness as the quality dimension. In our setting, the data provider offers an XML document, and sets both the price of the document and a weight to each node of the document, depending on its potential worth. The data consumer proposes a price. If the proposed price is lower than that of the entire document, then the data consumer receives a sample, i.e., a random rooted subtree of the document whose selection depends on the discounted price and the weight of nodes. By requesting several samples, the data consumer can iteratively explore the data in the document. We show that the uniform random sampling of a rooted subtree with prescribed weight is unfortunately intractable. However, we are able to identify several practical cases that are tractable. The first case is uniform random sampling of a rooted subtree with prescribed size; the second case restricts to binary weights. For both these practical cases we present polynomial-time algorithms and explain how they can be integrated into an iterative exploratory sampling approach.
\end{abstract}

\section{Introduction}

There are three kinds of actors in a data market: data consumers, data providers, and data market owners [14]. A data provider brings data to the market and sets prices on the data. A data consumer buys data from the market and pays for it. The owner is the broker between providers and consumers, who negotiates pricing schemes with data providers and manages transactions to trade data.

In most of the data pricing literature [4-6,9], data prices are prescribed and not negotiable, and give access to the best data quality that the provider can achieve. Yet, data quality is an important axis which should be used to price documents in data markets. Wang et al. $[15,18]$ define dimensions to assess data quality following four categories: intrinsic quality (believability, objectivity, accuracy, reputation), contextual quality (value-added, relevancy, timeliness, ease 
of operation, appropriate amount of data, completeness), representational quality (interpretability, ease of understanding, concise representation, consistent representation), and accessibility quality (accessibility, security).

In this paper, we focus on contextual quality and propose a data pricing scheme for XML trees such that completeness can be traded for discounted prices. This is in contrast to our previous work [17] where the accuracy of relational data is traded for discounted prices. Wang et al. [15,18] define completeness as "the extent to which data includes all the values, or has sufficient breadth and depth for the current task". We retain the first part of this definition as there is no current task defined in our setting. Formally, the data provider assigns, in addition to a price for the entire document, a weight to each node of the document, which is a function of the potential worth of this node: a higher weight is given to nodes that contain information that is more valuable to the data consumer. We define the completeness of a rooted subtree of the document as the total weight of its nodes, divided by the total weight of the document. A data consumer can then offer to buy an XML document for less than the provider's set price, but then can only obtain a rooted subtree of the original document, whose completeness depends on the discount granted.

A data consumer may want to pay less than the price of the entire document for various reasons: first, she may not be able to afford it due to limited budget but may be satisfied by a fragment of it; second, she may want to explore the document and investigate its content and structure before purchasing it fully.

The data market owner negotiates with the data provider a pricing function, allowing them to decide the price of a rooted subtree, given its completeness (i.e., the weight). The pricing function should satisfy a number of axioms: the price should be non-decreasing with the weight, be bounded by the price of the overall document, and be arbitrage-free when repeated requests are issued by the same data consumer (arbitrage here refers to the possibility to strategize the purchase of data). Hence, given a proposed price by a data consumer, the inverse of the pricing function decides the completeness of the sample that should be returned. To be fair to the data consumer, there should be an equal chance to explore every possible part of the XML document that is worth the proposed price. Based on this intuition, we sample a rooted subtree of the XML document of a certain weight, according to the proposed price, uniformly at random.

The data consumer may also issue repeated requests as she is interested in this XML document and wants to explore more information inside in an iterative manner. For each repeated request, a new rooted subtree is returned. A principle here is that the information (document nodes) already paid for should not be charged again. Thus, in this scenario, we sample a rooted subtree of the XML document of a certain weight uniformly at random, without counting the weight of the nodes already bought in previously issued requests.

The present article brings the following contributions:

- We propose to realize the trade-off between quality and discount in data markets. We propose a framework for pricing the completeness of XML data, 
based on uniform sampling of rooted subtrees in weighted XML documents. (Section 3)

- We show that the general uniform sampling problem in weighted XML trees is intractable. In this light, we propose two restrictions: sampling based on the number of nodes, and sampling when weights are binary (i.e., weights are 0 or 1 ). (Section 4 )

- We show that both restrictions are tractable by presenting a polynomialtime algorithm for uniform sampling based on the size of a rooted subtree, or on 0/1-weights. (Section 5)

- We extend our framework to the case of repeated sampling requests where the data consumer is not charged twice the same nodes. Again, we obtain tractability when the weight of a subtree is its size. (Section 6)

\section{Related Work}

Data Pricing. The basic structure of data markets and different pricing schemes were introduced in [14]. The notion of "query-based" pricing was introduced in [4, $6]$ to define the price of a query as the price of the cheapest set of pre-defined views that can determine the query. It makes data pricing more flexible, and serves as the foundation of a practical data pricing system [5]. The price of aggregate queries has been studied in [9]. Different pricing schemes are investigated and multiple pricing functions are proposed to avoid several pre-defined arbitrage situations in [10]. However, none of the works above takes data quality into account, and those works do not allow the data consumer to propose a price less than that of the data provider, which is the approach that we study here.

The idea of trading off price for data quality has been explored in the context of privacy in [8], which proposes a theoretic framework to assign prices to noisy query answers. If a data consumer cannot afford the price of a query, she can choose to tolerate a higher standard deviation to lower the price. However, this work studies pricing on accuracy for linear relational queries, rather than pricing XML data on completeness. In [17], we propose a relational data pricing framework in which data accuracy can be traded for discounted prices. By contrast, this paper studies pricing for XML data, and proposes a tradeoff based on data completeness rather than accuracy.

Subtree/Subgraph Sampling. The main technical result of this paper is the tractability of uniform subtree sampling under a certain requested size. This question is related to the general topic of subtree and subgraph sampling, but, to our knowledge, it has not yet been adequately addressed.

Subgraph sampling works such as $[3,7,13,16]$ have proposed algorithms to sample small subgraphs from an original graph while attempting to preserve selected metrics and properties such as degree distribution, component distribution, average clustering coefficient and community structure. However, the distribution from which these random graphs are sampled is not known and cannot be guaranteed to be uniform. 
Other works have studied the problem of uniform sampling $[2,11]$. However, [2] does not propose a way to fix the size of the samples. The authors of [11] propose a sampling algorithm to sample a connected sub-graph of size $k$ under an approximately uniform distribution; note that this work provides no bound on the error relative to the uniform distribution.

Sampling approaches are used in $[19,12]$ to estimate the selectivity of XML queries (containment join and twig queries, respectively). Nevertheless, the samples in [19] are specific to containment join queries, while those in [12] are representatives of the XML document for any twig queries. Neither of those works controls the distribution from which the subtrees are sampled.

In [1], Cohen and Kimelfeld show how to evaluate a deterministic tree automaton on a probabilistic XML document, with applications to sampling possible worlds that satisfy a given constraint, e.g., expressed in monadic second-order logic and then translated into a tree automaton. Note that the translation of constraints to tree automata itself is not tractable; in this respect, our approach can be seen as a specialization of [1] to the simpler case of fixed-size or fixed-weight tree sampling, and as an application of it to data pricing.

\section{Pricing Function and Sampling Problem}

This paper studies data pricing for tree-shaped documents. Let us first formally define the terminology that we use for such documents.

We consider trees that are unordered, directed, rooted, and weighted. Formally, a tree $t$ consists of a set of nodes $\mathrm{V}(t)$ (which are assumed to carry unique identifiers), a set of edges $\mathrm{E}(t)$, and a function w mapping every node $n \in \mathrm{V}(t)$ to a non-negative rational number $\mathrm{w}(n)$ which is the weight of node $n$. We write $\operatorname{root}(t)$ for the root node of $t$. Any two nodes $n_{1}, n_{2} \in \mathrm{V}(t)$ such that $\left(n_{1}, n_{2}\right) \in \mathrm{E}(t)$ are in a parent-child relationship, that is, $n_{1}$ is the parent of $n_{2}$ and $n_{2}$ is a child of $n_{1}$.

By children $(n)$, we represent the set of nodes that have parent $n$. A tree is said to be binary if each node of the tree has at most two children, otherwise it is unranked. Throughout this paper, for ease of presentation, we may call such trees "XML documents".

We now introduce the notion of rooted subtree of an XML document:

Definition 1. (Subtree, rooted subtree) $A$ tree $t^{\prime}$ is a subtree of a tree $t$ if $\mathrm{V}\left(t^{\prime}\right) \subseteq \mathrm{V}(t)$ and $\mathrm{E}\left(t^{\prime}\right) \subseteq \mathrm{E}(t)$. A rooted subtree $t^{\prime}$ of a tree $t$ is a subtree of $t$ such that $\operatorname{root}(t)=\operatorname{root}\left(t^{\prime}\right)$. We name it $\boldsymbol{r}$-subtree for short. The weight function for a subtree $t^{\prime}$ of a tree $t$ is always assumed to be the restriction of the weight function for $t$ on the nodes in $t^{\prime}$.

For technical reasons, we also sometimes talk of the empty subtree that contains no node.

Example 1. Figure 1 presents two example trees. The nodes $\left\{n_{0}, n_{2}, n_{5}\right\}$, along with the edges connecting them, form an r-subtree of the tree in Figure 1(a). 


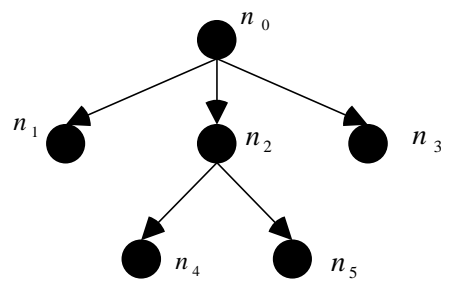

(a) A tree

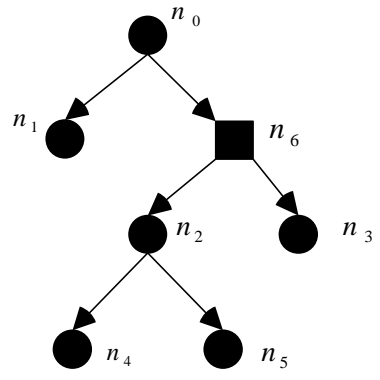

(b) A binary tree

Fig. 1. Two example trees (usage of the square node will be introduced in Section 5.2)

Likewise, the nodes $\left\{n_{2}, n_{4}, n_{5}\right\}$ and the appropriate edges form a subtree of that tree (but not an r-subtree). The tree of Figure 1(b) is a binary tree (ignore the different shapes of the nodes for now).

We now present our notion of data quality, by defining the completeness of an r-subtree, based on the weight function of the original tree:

Definition 2. (Weight of a tree) For a node $n \in \mathrm{V}(t)$, we define inductively weight $(n)=\mathrm{w}(n)+\sum_{\left(n, n^{\prime}\right) \in \mathrm{E}(t)}$ weight $\left(n^{\prime}\right)$. With slight abuse of notation, we note weight $(t)=$ weight $(\operatorname{root}(t))$ as the weight of $t$.

Definition 3. (Completeness of an r-subtree) Let $t$ be a tree. Let $t^{\prime}$ be an $r$ subtree of $t$. The completeness of $t^{\prime}$ with respect to $t$ is $c_{t}\left(t^{\prime}\right)=\frac{\text { weight }\left(t^{\prime}\right)}{\text { weight }(t)}$. It is obvious that $c_{t}\left(t^{\prime}\right) \in[0,1]$.

We study a framework for data markets where the data consumer can buy an incomplete document from the data provider while paying a discounted price. The formal presentation of this framework consists of three parts:

1. An XML document $t$.

2. A pricing function $\varphi_{t}$ for $t$ whose input is the desired completeness for an rsubtree of the XML document, and whose value is the price of this r-subtree. Hence, given a proposed price $p r_{0}$ by a data consumer, the completeness of the returned r-subtree is decided by $\varphi_{t}^{-1}\left(p r_{0}\right)$.

3. An algorithm to sample an r-subtree of the XML document uniformly at random among those of a given completeness.

We study the question of the sampling algorithm more in detail in subsequent sections. For now, we focus on the pricing function, starting with a formal definition:

Definition 4. (Pricing function) The pricing function for a tree $t$ is a function $\varphi_{t}:[0,1] \rightarrow \mathbb{Q}^{+}$. Its input is the completeness of an r-subtree $t^{\prime}$ and it returns the price of $t^{\prime}$, as a non-negative rational. 
A healthy data market should impose some restrictions on $\varphi_{t}$, such as:

Non-decreasing. The more complete an $\mathrm{r}$-subtree is, the more expensive it should be, i.e., $c_{1} \geqslant c_{2} \Rightarrow \varphi_{t}\left(c_{1}\right) \geqslant \varphi_{t}\left(c_{2}\right)$.

Arbitrage-free. Buying an r-subtree of completeness $c_{1}+c_{2}$ should not be more expensive than buying two subtrees with respective completeness $c_{1}$ and $c_{2}$, i.e., $\varphi_{t}\left(c_{1}\right)+\varphi_{t}\left(c_{2}\right) \geqslant \varphi_{t}\left(c_{1}+c_{2}\right)$. In other words, $\varphi_{t}$ should be subadditive. This property is useful when considering repeated requests, studied in Section 6.

Minimum and maximum bound. We should have $\varphi_{t}(0)=p r_{\min }$ and $\varphi_{t}(1)=$ $p r_{t}$, where $p r_{\text {min }}$ is the minimum cost that a data consumer has to pay using the data market and $p r_{t}$ is the price of the whole tree $t$. Note that by the non-decreasing character of $\varphi_{t}, p r_{t} \geqslant p r_{\min } \geqslant 0$.

All these properties can be satisfied, for instance, by functions of the form $\varphi_{t}(c)=\left(p r_{t}-p r_{\text {min }}\right) c^{p}+p r_{\text {min }}$ where $p \leqslant 1$; however, if $p>1$, the arbitrage-free property is violated.

Given a proposed price $p r_{0}$ by a data consumer, $\varphi_{t}^{-1}\left(p r_{0}\right)$ is the set of possible corresponding completeness values. Note that $\varphi_{t}^{-1}$ is a relation and may not be a function; $\varphi_{t}^{-1}$ is a function if different completeness values correspond to different prices. Once a completeness value $c \in \varphi_{t}^{-1}\left(p r_{0}\right)$ is chosen, the weight of the returned r-subtree is fixed as $c \times$ weight $(t)$.

Therefore, in the rest of the paper, we consider the problem of uniform sampling an r-subtree with prescribed weight (instead with prescribed completeness). Let us now define the problem that should be solved by our sampling algorithm:

Definition 5. (Sampling problem) The problem of sampling an r-subtree, given a tree $t$ and a weight $k$, is to sample an $r$-subtree $t^{\prime}$ of $t$, such that weight $\left(t^{\prime}\right)=k$, uniformly at random, if one exists, or fail if no such r-subtree exists.

\section{Tractability}

\subsection{Intractability of the Sampling Problem}

We now turn to the question of designing an algorithm to solve the sampling problem defined in the previous section. Sadly, it can be shown that this problem is already NP-hard in the general formulation that we gave.

Proposition 1. Given a tree $t$ and a weight $x$, it is NP-hard to sample an $r$-subtree of $t$ of weight $x$ uniformly at random.

Proof. Consider the simpler problem of deciding if such an r-subtree of $t$ exists. Further restrict $t$ such that $\mathrm{w}(n)=0$ unless $n$ is a leaf. In this case, deciding whether there exists an $\mathrm{r}$-subtree of weight $x$ is equivalent to the subset-sum problem, which is known to be NP-complete. Now there is a PTIME-reduction from this existence problem to the sampling problem, as an algorithm for sampling can be used to decide whether there exists an r-subtree of the desired weight (the algorithm returns one such) or if none exists (the algorithm fails). 


\subsection{Tractable Cases}

We now define restricted variants of the sampling problem where the weight function is assumed to be of a certain form. In the next section, we show that sampling for these variants can be performed in PTIME.

Unweighted Sampling. In this setting, we take the weight function $\mathrm{w}(n)=1$ for all $n \in \mathrm{V}(t)$. Hence, the weight of a tree $t$ is actually the number of nodes in $t$, i.e., its size, which we write size $(t)$.

In this case, the hardness result of Proposition 1 does not apply anymore. However, sampling an r-subtree with prescribed size uniformly at random is still not obvious to do, as the following example shows:

Example 2. Consider the problem of sampling an r-subtree $t^{\prime}$ of size 3 from the tree $t$ in Figure 1(a). We can enumerate all such r-subtrees: $\left\{n_{0}, n_{1}, n_{2}\right\}$, $\left\{n_{0}, n_{1}, n_{3}\right\},\left\{n_{0}, n_{2}, n_{3}\right\},\left\{n_{0}, n_{2}, n_{4}\right\}$ and $\left\{n_{0}, n_{2}, n_{5}\right\}$, and choose one of them at random with probability $\frac{1}{5}$. However, as the number of r-subtrees may be exponential in the size of the document in general, we cannot hope to perform this approach in PTIME. Observe that it is not easy to build a random r-subtree node by node: it is clear that node $n_{0}$ must be included, but then observe that we cannot decide to include $n_{1}, n_{2}$, or $n_{3}$, uniformly at random. Indeed, if we do this, our distribution on the r-subtrees will be skewed, as $n_{1}$ (or $n_{3}$ ) occurs in $\frac{2}{5}$ of the outcomes whereas $n_{2}$ occurs in $\frac{4}{5}$ of them. Intuitively, this is because there are more ways to choose the next nodes when $n_{2}$ is added, than when $n_{1}$ or $n_{3}$ are added.

We show in the next section that this problem can be solved in PTIME.

0/1-weights Sampling. In this more general problem variant, we require that $\mathrm{w}(n) \in\{0,1\}$ for all $n \in \mathrm{V}(t)$, i.e., the weight is binary.

We show in Section 5.3 that this problem can also be solved in PTIME using an adaptation of the unweighted sampling algorithm.

\section{Algorithms for Tractable Uniform Sampling}

In this section, we present a PTIME algorithm for the unweighted sampling problem, namely the problem of sampling an r-subtree of size $k$ from an XML document, uniformly at random.

We first describe the algorithm for the case of binary trees, in Section 5.1. Next, we adapt the algorithm in Section 5.2 to show how to apply it to arbitrary trees. Last, we study the more general case of 0/1-weights in Section 5.3, showing that the algorithm for unweighted sampling can be adapted to solve this problem. 

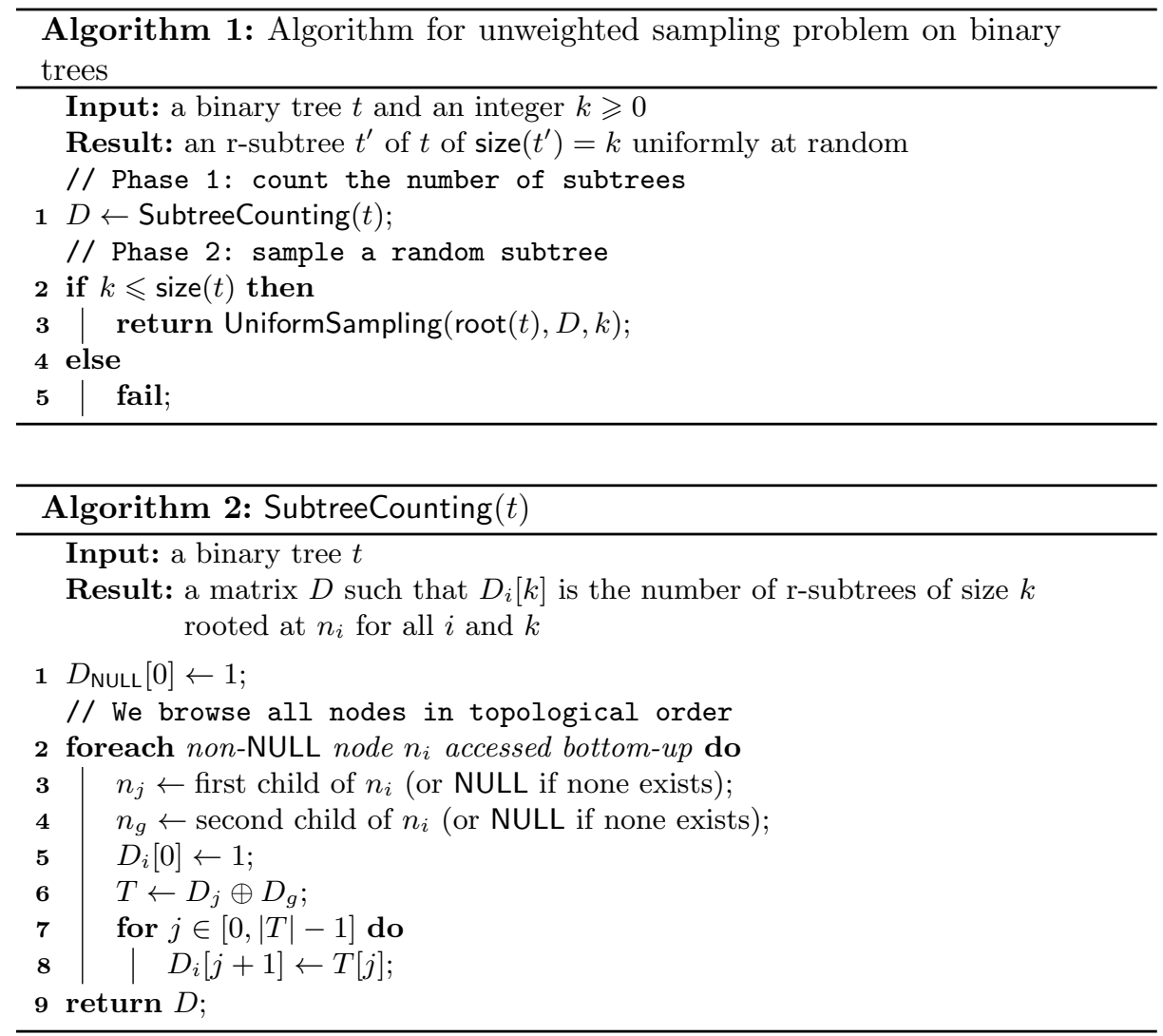

\subsection{Unweighted Sampling for Binary Trees}

In this section, we provide an algorithm which proves the following theorem:

Theorem 1. The unweighted sampling problem for binary trees can be solved in PTIME.

Our general algorithm to solve this problem is given as Algorithm 1. The algorithm has two phases, which we study separately in what follows. For simplicity, whenever we discuss binary trees in this section, we will add special NULL children to every node of the tree (except NULL nodes themselves), so that all nodes, including leaf nodes, have exactly two children (which may be NULL). This will simplify the presentation of the algorithms.

First phase: Subtree Counting (Algorithm 2). We start by computing a matrix $D$ such that, for every node $n_{i}$ of the input tree $t$ and any value $0 \leqslant k \leqslant \operatorname{size}(t), D_{i}[k]$ is the number of subtrees of size $k$ rooted at node $n_{i}$. We do so with Algorithm 2 which we now explain in detail. 
There is only one subtree rooted at the special NULL node, namely the empty subtree, with size 0 , which provides the base case of the algorithm (line 1). Otherwise, we compute $D_{i}$ for a node $n_{i}$ from the values $D_{j}$ and $D_{g}$ of $D$ for its children $n_{j}$ and $n_{g}$ (which may be NULL); those values have been computed before because nodes are considered following a topological ordering, in a bottom-up fashion.

Intuitively, the only r-subtree of size 0 is the empty subtree, hence we must have $D_{i}[0]=1$ (line 5). Otherwise, any r-subtree of size $k>0$ rooted at $n_{i}$ is obtained by retaining $n_{i}$, and choosing two r-subtrees $t_{j}$ and $t_{g}$, respectively rooted at $n_{j}$ and $n_{g}$ (the children of $n_{i}$ ), such that size $\left(t_{j}\right)+\operatorname{size}\left(t_{g}\right)=k-1$ (which accounts for the size of the additional node $n_{i}$ ). The number of such choices is computed by the convolution of $D_{j}$ and $D_{g}$ in line 6 , defined as:

$$
\text { For } 0 \leqslant p \leqslant\left|D_{j}\right|+\left|D_{g}\right|-2, \quad\left(D_{j} \oplus D_{g}\right)[p]:=\sum_{m=\max \left(0, p-\left|D_{g}\right|-1\right)}^{\min \left(p,\left|D_{j}\right|-1\right)} D_{j}[m] \times D_{g}[p-m] \text {. }
$$

Lines 7 and 8 account for the size of the retained node $n_{i}$.

Example 3. Let $t$ be the tree presented in Figure 1(b) (again, ignore the different shapes of nodes for now). Starting from the leaf nodes, we compute $D_{1}=D_{4}=$ $D_{5}=D_{3}=(1,1)$, applying lines 5 to 8 on $D_{\mathrm{NULL}} \oplus D_{\mathrm{NULL}}=(1)$. This means that there are two r-subtrees rooted at leaf nodes: the empty subtree, and the subtree with just that leaf.

Now, when computing $D_{2}$, we first convolve $D_{4}$ and $D_{5}$ to get the numbers of pairs of r-subtrees of different sizes at $\left\{n_{4}, n_{5}\right\}$, i.e., $D_{4} \oplus D_{5}=(1,2,1)$, so that $D_{2}=(1,1,2,1)$. When computing $D_{6}$, we first compute $D_{2} \oplus D_{3}=(1,2,3,3,1)$, so that $D_{6}=(1,1,2,3,3,1)$. Finally, $D_{0}=(1,1,2,3,5,6,4,1)$.

We now state the correctness and running time of this algorithm.

Lemma 1. Algorithm 2 terminates in time polynomial in size $(t)$ and returns $D$ such that, for every $i$ and $k, D_{i}[k]$ is the number of $r$-subtrees of size $k$ rooted at node $n_{i}$.

Proof. Let us first prove the running time. All arrays under consideration have size at most $n(n=\operatorname{size}(t))$, so computing the convolution sum of two such arrays is in time $O\left(n^{2}\right)$ (computing each value of the convolution sum is in time $O(n)$ ). The number of convolution sums to compute overall is $O(n)$, because each array $D_{i}$ occurs in exactly one convolution sum. The overall running time is $O\left(n^{3}\right)$.

Let us now show correctness. We proceed by induction on the node $n_{i}$ to prove the claim for every $k$. The base case is the NULL node, whose correctness is straightforward. Let us prove the induction step. Let $n_{i}$ be a node, and assume by induction that $D_{j}\left[k^{\prime}\right]$ is correct for every $k^{\prime}$ for every child $n_{j}$ of $n_{i}$. Let us fix $k$ and show that $D_{i}[k]$ is correct. To select an r-subtree at $n_{i}$, either $k=0$ and there is exactly one possibility (the empty subtree), or $k>0$ and the number of possibilities is the number of ways to select a set of r-subtrees at the children of $n_{i}$ so that their size sum to $k-1$. This is the role of lines 5 to 8 . Now, to 


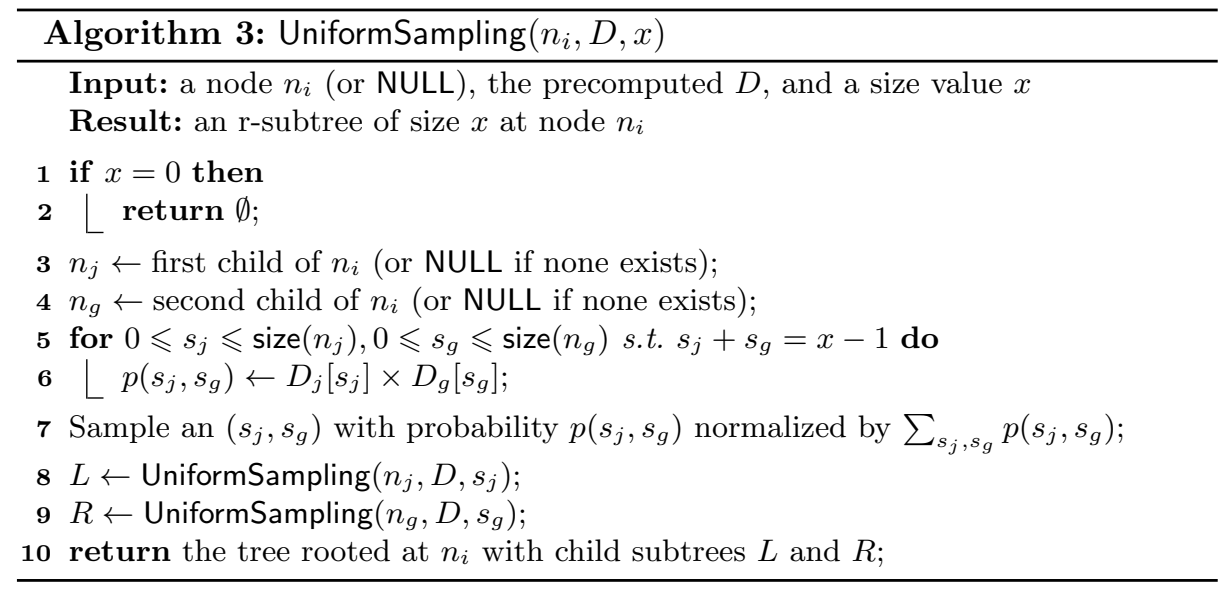

enumerate the ways of choosing r-subtrees at children of $n_{i}$ whose size sum to $k-1$, we can first decide the size of the selected r-subtree for each child: the ways to assign such sizes form a partition of the possible outcomes, so the number of outcomes is the sum, over all such assignments of r-subtree sizes to children, of the number of outcomes for this assignment. Now, for a fixed assignment, the subtrees rooted at each children are chosen independently, so the number of outcomes for a fixed assignment is the product of the number of outcomes for the given size for each child, which by induction hypothesis is correctly reflected by the corresponding $D_{j}\left[k^{\prime}\right]$. Hence, for a given $k$, either $k=0$, or $k>0$, in which case $D_{i}[k]$ is $\left(D_{j} \oplus D_{g}\right)[k-1]$ by lines 5 to 8 , which sums, over all possible subtree size assignments, the number of choices for this subtree size assignment. Hence, by induction, we have shown the desired claim.

Second phase: Uniform Sampling (Algorithm 3). In the second phase of Algorithm 1, we sample an r-subtree from $t$ in a recursive top-down manner, based on the matrix $D$ computed by Algorithm 2. Our algorithm to perform this uniform sampling is Algorithm 3. The basic idea is that to sample an rsubtree rooted at a node $n_{i}$, we decide on the size of the subtrees rooted at each child node, biased by the number of outcomes as counted in $D$, and then sample r-subtrees of the desired size recursively.

Let us now explain Algorithm 3 in detail.

If $x=0$, we must return the empty tree (lines 2 and 2). Otherwise we return $n_{i}$ and subtrees $t_{j}$ and $t_{g}$ rooted at the children $n_{j}$ and $n_{g}$ of $n_{i}$. We first decide on the size $s_{j}$ and $s_{g}$ of $t_{j}$ and $t_{g}$ (lines 6 to 7 ) before sampling recursively a subtree of the prescribed size, uniformly at random, and returning it.

The possible size pairs $\left(s_{j}, s_{g}\right)$ must satisfy the following conditions to be possible choices for the sizes of the subtrees $t_{j}$ and $t_{g}$ :

1. $0 \leqslant s_{j} \leqslant \operatorname{size}\left(n_{j}\right)$ and $0 \leqslant s_{g} \leqslant \operatorname{size}\left(n_{g}\right)$ (of course size $(\mathrm{NULL})=0$ )

2. $s_{j}+s_{g}=x-1$ (which accounts for node $n_{i}$ ) 
Intuitively, to perform a uniform sampling, we now observe that the choice of the size pair $\left(s_{j}, s_{g}\right)$ partitions the set of outcomes. Hence, the probability that we select one size pair should be proportional to the number of possible outcomes for this pair, namely, the number of r-subtrees $t_{j}$ and $t_{g}$ such that size $\left(t_{j}\right)=s_{j}$ and size $\left(t_{g}\right)=s_{g}$. We compute this from $D_{j}$ and $D_{g}$ (line 6) by observing that the number of pairs $\left(t_{j}, t_{g}\right)$ is the product of the number of choices for $t_{j}$ and for $t_{g}$, as every combination of choices is possible.

Example 4. Follow Example 3. Assume we want to sample an r-subtree $t^{\prime}$ of $\operatorname{size}\left(t^{\prime}\right)=3$ uniformly. We first call UniformSampling $\left(n_{0}, 3\right)$. We have to return $n_{0}$. Now $n_{0}$ has two children, $n_{1}$ and $n_{6}$. The possible size pairs are $(0,2)$ and $(1,1)$, with respective (unnormalized) probabilities $p(0,2)=D_{1}[0] \times D_{6}[2]=$ $1 \times 2=2$ and $p(1,1)=D_{1}[1] \times D_{6}[1]=1 \times 1=1$. The normalized probabilities are therefore $\frac{2}{3}$ and $\frac{1}{3}$. Assume that we choose $(0,2)$. We now call recursively UniformSampling $\left(n_{1}, 0\right)$ and UniformSampling $\left(n_{6}, 2\right)$.

UniformSampling $\left(n_{1}, 0\right)$ returns $\emptyset$. We proceed to UniformSampling $\left(n_{6}, 2\right)$. We have to return $n_{6}$. Now $n_{6}$ has two children, $n_{2}$ and $n_{3}$. The possible size pairs for this call are $(1,0)$ and $(0,1)$ with probabilities $\frac{1}{2}$ and $\frac{1}{2}$. Assume that we choose $(1,0)$. We now call recursively UniformSampling $\left(n_{2}, 1\right)$ and UniformSampling $\left(n_{3}, 0\right)$.

UniformSampling $\left(n_{3}, 0\right)$ returns $\emptyset$. We proceed to UniformSampling $\left(n_{2}, 1\right) . n_{2}$ is selected. $n_{2}$ has two children, $n_{4}$ and $n_{5}$. There is only one possible size pair for this call $(0,0)$ with probability 1 . We can only choose $(0,0)$ and call UniformSampling $\left(n_{4}, 0\right)$ (which results in $\emptyset$ ) and UniformSampling $\left(n_{5}, 0\right)$ (which results in $\emptyset$ ). Hence, the end result is the r-subtree whose nodes are $\left\{n_{0}, n_{6}, n_{2}\right\}$ (and whose edges can clearly be reconstituted in PTIME from $t$ ).

We now show the tractability and correctness of Algorithm 3.

Lemma 2. For any tree $t$, node $n_{i} \in \mathrm{V}(t)$ and integer $0 \leqslant x \leqslant \operatorname{size}\left(n_{i}\right)$, given $D$ computed by Algorithm 2, UniformSampling $\left(n_{i}, D, x\right)$ terminates in polynomial time and returns an r-subtree of size $x$ rooted at $n_{i}$, uniformly at random (i.e., solves the unweighted sampling problem for binary trees).

Proof. Let us first prove the complexity claim. On every node $n_{i}$ of the binary tree $t$, the number of possibilities to consider is linear in the tree size because every node has exactly two children, and for each possibility the number of operations performed is constant (assuming that drawing a number uniformly at random is constant-time). The overall running time is quadratic, i.e., $O\left(\operatorname{size}(t)^{2}\right)$.

We now show correctness by induction on $n_{i}$. The base case is $n_{i}=$ NULL, in which case we must have $x=0$ and we correctly return $\emptyset$. Otherwise, if $n_{i}$ is not NULL, either $x=0$ and we correctly return $\emptyset$, or $x>0$ and, as in the proof of Lemma 1, the set of possible outcomes of the sampling process is partitioned by the possible assignments, and only the valid ones correspond to a non-empty set of outcomes. Hence, we can first choose a size pair, weighted by the proportion of outcomes which are outcomes for that assignment, and then choose an outcome for this pair. Now, observe that, by Lemma 1, D correctly represents the number of outcomes for each child of $n_{i}$, so that our computation 
of $p$ (which mimics that of Algorithm 2) correctly represents the proportion of outcomes that are outcomes for every size pair. We then choose an assignment according to $p$, and then observe that choosing an outcome for this assignment amounts to choosing an outcome for each child of $n_{i}$ whose size is given by the assignment. By induction hypothesis, this is precisely what the recursive calls to UniformSampling $\left(n_{i}, D, x\right)$ perform. This concludes the proof.

\subsection{Algorithm for Sampling an Unranked Tree}

In this section, we show that the algorithm of the previous section can be adapted so that it works on arbitrary unranked trees, not just binary trees.

We first observe that the straightforward generalization of Algorithm 1 to trees of arbitrary arity, where assignments and convolutions are performed for all children, is still correct. However, it would not run in polynomial time anymore as there would be a potentially exponential number of size pairs to consider.

Fortunately, there is still hope to avoid enumerating the size pairs over all the children, thanks to the associativity of convolution sum. Informally, assume we have three children $\left\{n_{1}, n_{2}, n_{3}\right\}$, we do the following: we treat $\left\{n_{1}\right\}$ as a group and $\left\{n_{2}, n_{3}\right\}$ as the second group, then enumerate size pairs over $\left\{n_{1}\right\}$ and $\left\{n_{2}, n_{3}\right\}$; once a size pair, in which a positive integer is assigned to $\left\{n_{2}, n_{3}\right\}$, is selected, we can treat $\left\{n_{2}\right\}$ and $\left\{n_{3}\right\}$ as new groups and enumerate size pairs over $\left\{n_{2}\right\}$ and $\left\{n_{3}\right\}$. This strategy can be implemented by transforming the original tree to a binary tree. From this intuition, we now state our result:

Theorem 2. The unweighted sampling problem can be solved in PTIME, for arbitrary unranked trees.

Proof (Sketch). The proof proceeds by encoding arbitrary trees to encoded trees, that are binary trees whose nodes are either regular nodes or dummy nodes. Intuitively, the encoding operation replaces sequences of more than two children by a hierarchy of dummy nodes representing those children; replacing dummy nodes by the sequence of their children yields back the original tree. The encoding is illustrated in Figure 1, where the tree in Figure 1(b) is the encoded tree of the one in Figure 1(a) (dummy nodes are represented as squares).

It can then be shown that, up to the question of keeping or deleting the dummy nodes with no regular descendants (we call them bottommost), there is a bijection between r-subtrees in the original tree and r-subtrees in the encoded tree. Hence, we can solve the unweighted sampling problem by choosing an $\mathrm{r}-$ subtree in the encoded tree with the right number of regular nodes, uniformly at random, and imposing the choice of keeping bottommost dummy nodes.

There only remains to adapt Algorithms 2 and 3 to run correctly on encoded trees, that is, mananging dummy nodes correctly.

We change Algorithm 2 by replacing lines 5 to 8 with $D_{i} \leftarrow D_{j} \oplus D_{g}$ when $n_{i}$ is a dummy node (as it must always be kept, and does not increase the size of the r-subtree).

We change Algorithm 3 by imposing at line 2 the condition that $n_{i}$ is either NULL or a regular node (otherwise we cannot return $\emptyset$ as we must keep dummy 
nodes). Also, we change line 6 so that, when node $n_{i}$ is a dummy node, we require $s_{j}+s_{g}=x$ (rather than $x-1$ ), as we do not count the dummy node in the size of the resulting subtree.

The correctness and running time of the modified algorithms can be proved by straightforward adaptations of Lemma 1 and Lemma 2 .

\subsection{Uniform Sampling under 0/1-weights}

Our tractability result extends to trees with binary weights:

Theorem 3. The 0/1-weights sampling problem can be solved in PTIME, for arbitrary unranked trees.

Proof (Sketch). The proof proceeds by modifying the unweighted sampling algorithm to handle nodes of weight 0 (in addition to the modifications described in the previous section).

In Algorithm 2, when $n_{i}$ is a weight- 0 node, lines 7 to 8 must be replaced by $j \in[1,|T|-1], D_{i}[j] \leftarrow T[j]$ (the node has no weight); line 5 is removed and set $D_{i}[0] \leftarrow 1+T[0]$ (we can keep or discard the weight-0 node, unlike the dummy nodes of the previous section).

In Algorithm 3, when $x=0$ and $n_{i}$ is a weight-0 node, the empty subtree should be returned with probability $\frac{1}{D_{i}[0]}$; otherwise, continue the execution of the algorithm to return a random non-empty r-subtree of size 0 . This is because the introduction of weight- 0 nodes, unlike weight- 1 and dummy nodes, leads to multiple ways to sample a r-subtree of size 0 . If $n_{i}$ is a weight- 0 node, line 6 should be changed to require $s_{j}+s_{g}=x$ rather than $x-1$, as for dummy nodes.

The correctness and running time of the modified algorithms can be proved by straightforward adaptations of Lemmas 1 and 2 .

\section{$6 \quad$ Repeated Requests}

In this section, we consider the more general problem where the data consumer requests a completion of a certain price to data that they have already bought. The motivation is that, after having bought incomplete data, the user may realize that they need additional data, in which case they would like to obtain more incomplete data that is not redundant with what they already have.

A first way to formalize the problem is as follows, where data is priced according to a known subtree (provided by the data consumer) by considering that known nodes are free (but that they may or may not be returned again).

Definition 6. The problem of sampling an r-subtree of weight $k$ in a tree $t$ conditionally to an r-subtree $t^{\prime}$ is to sample an r-subtree $t^{\prime \prime}$ of $t$ uniformly at random, such that weight $\left(t^{\prime \prime}\right)-\sum_{n \in\left(\mathrm{V}\left(t^{\prime}\right) \cap \mathrm{V}\left(t^{\prime \prime}\right)\right)} \mathrm{w}(n)=k$.

An alternative is to consider that we want to sample an extension of a fixed size to the whole subtree, so that all known nodes are part of the output: 
Definition 7. The problem of sampling an r-subtree of weight $k$ in a tree $t$ that extends an r-subtree $t^{\prime}$ is to sample an r-subtree $t^{\prime \prime}$ of $t$ uniformly at random, such that (1) $t^{\prime}$ is an r-subtree of $t^{\prime \prime}$; (2) weight $\left(t^{\prime \prime}\right)$ - weight $\left(t^{\prime}\right)=k$.

Note that those two formulations are not the same: the first one does not require the known part of the document to be returned, while the second one does. While it may be argued that the resulting outcomes are essentially equivalent (as they only differ on parts of the data that are already known to the data consumer), it is important to observe that they define different distributions: though both problems require the sampling to be uniform among their set of outcomes, the additional possible outcomes of the first definition means that the underlying distribution is not the same.

As the uniform sampling problem for r-subtrees can be reduced to either problem by setting $t^{\prime}$ to be the empty subtree, the NP-hardness of those two problems follows from Proposition 1. However, we can show that, in the unweighted case, those problems are tractable, because they reduce to the 0/1-weights sampling problem which is tractable by Theorem 3 :

Proposition 2. The problem of sampling an r-subtree of weight $k$ in a tree $t$ conditionally to an r-subtree $t^{\prime}$ can be solved in PTIME if $t$ is unweighted. The same holds for the problem of sampling that extends another r-subtree.

Proof (Sketch). For the problem of Definition 6, set the weight of the nodes of $t^{\prime}$ in $t$ to be zero (the intuition is that all the known nodes are free). The problem can then be solved by applying Theorem 3 .

For the problem of Definition 7, set the weight of the nodes of $t^{\prime}$ in $t$ to be zero but we have to ensure that weight-0 nodes are always returned. To do so, we adapt Theorem 3 by handling weight-0 nodes in the same way as handling dummy nodes in the previous section.

\section{Conclusion}

We proposed a framework for a data market in which data quality can be traded for a discount. We studied the case of XML documents with completeness as the quality dimension. Namely, a data provider offers an XML document, and sets both the price and weights of nodes of the document. The data consumer proposes a price but may get only a sample if the proposed price is lower than that of the entire document. A sample is a rooted subtree of prescribed weight, as determined by the proposed price, sampled uniformly at random.

We proved that if nodes in the XML document have arbitrary non-negative weights, the sampling problem is intractable. We identified tractable cases, namely the unweighted sampling problem and 0/1-weights sampling problem, for which we devised PTIME algorithms. We proved the time complexity and correctness of the algorithms. We also considered repeated requests and provided PTIME solutions to the unweighted cases. 
The more general issue that we are currently investigating is that of sampling rooted subtrees uniformly at random under more expressive conditions than size restrictions or 0/1-weights. In particular, we intend to identify the tractability boundary to describe the class of tree statistics for which it is possible to sample r-subtrees in PTIME under a uniform distribution.

Acknowledgment. This work is supported by the French Ministry of European and Foreign Affairs under the STIC-Asia program, CCIPX project.

\section{References}

1. S. Cohen, B. Kimelfeld, and Y. Sagiv. Running tree automata on probabilistic XML. In PODS, pages 227-236, 2009.

2. M. R. Henzinger, A. Heydon, M. Mitzenmacher, and M. Najork. On near-uniform URL sampling. Computer Networks, 33(1-6), 2000.

3. C. Hübler, H.-P. Kriegel, K. Borgwardt, and Z. Ghahramani. Metropolis algorithms for representative subgraph sampling. In $I C D M, 2008$.

4. P. Koutris, P. Upadhyaya, M. Balazinska, B. Howe, and D. Suciu. Query-based data pricing. In PODS, 2012.

5. P. Koutris, P. Upadhyaya, M. Balazinska, B. Howe, and D. Suciu. QueryMarket demonstration: Pricing for online data markets. PVLDB, 5(12), 2012.

6. P. Koutris, P. Upadhyaya, M. Balazinska, B. Howe, and D. Suciu. Toward practical query pricing with QueryMarket. In SIGMOD, 2013.

7. J. Leskovec and C. Faloutsos. Sampling from large graphs. In SIGKDD, 2006.

8. C. Li, D. Y. Li, G. Miklau, and D. Suciu. A theory of pricing private data. In ICDT, 2013.

9. C. Li and G. Miklau. Pricing aggregate queries in a data marketplace. In WebDB, 2012.

10. B.-R. Lin and D. Kifer. On arbitrage-free pricing for general data queries. PVLDB, $7(9): 757-768,2014$.

11. X. Lu and S. Bressan. Sampling connected induced subgraphs uniformly at random. In $S S D B M, 2012$.

12. C. Luo, Z. Jiang, W.-C. Hou, F. Yu, and Q. Zhu. A sampling approach for XML query selectivity estimation. In EDBT, 2009.

13. A. S. Maiya and T. Y. Berger-Wolf. Sampling community structure. In $W W W$, 2010.

14. A. Muschalle, F. Stahl, A. Loser, and G. Vossen. Pricing approaches for data markets. In BIRTE, 2012.

15. L. Pipino, Y. W. Lee, and R. Y. Wang. Data quality assessment. Commun. ACM, 45(4), 2002.

16. B. F. Ribeiro and D. F. Towsley. Estimating and sampling graphs with multidimensional random walks. In Internet Measurement Conference, 2010.

17. R. Tang, D. Shao, S. Bressan, and P. Valduriez. What you pay for is what you get. In DEXA (2), 2013.

18. R. Y. Wang and D. M. Strong. Beyond accuracy: What data quality means to data consumers. J. of Management Information Systems, 12(4), 1996.

19. W. Wang, H. Jiang, H. Lu, and J. X. Yu. Containment join size estimation: Models and methods. In SIGMOD, 2003. 